\title{
Net Degrees of Freedom of Decomposition Schemes for the MIMO IC with Delayed CSIT
}

\author{
Yohan Lejosne and Dirk Slock \\ EURECOM $^{\dagger}$, Mobile Communications Dept. \\ Campus SophiaTech, 450 route des Chappes \\ 06410 Biot Sophia Antipolis, FRANCE \\ Email: \{yohan.lejosne,dirk.slock\}@eurecom.fr
}

\author{
Yi Yuan-Wu \\ Orange Labs*, IMT/OLN \\ 38-40 Rue du General Leclerc \\ 92794 Issy Moulineaux Cedex 9, FRANCE \\ Email: \{yohan.lejosne, yi.yuan\}@orange.com
}

\begin{abstract}
Most techniques designed for the multiple-input multiple-output (MIMO) Interference Channel (IC) require accurate current channel state information at the transmitter (CSIT) which is not a realistic assumption because of feedback delay. We evaluate the net degrees of freedom (DoF) that different schemes can be expected to reach in a realistic system by taking into account the time and the cost of CSIT acquisition (training and feedback). A recent variant of ergodic interference alignment (IA) clearly outperforms the other schemes as its robustness to feedback delays proves to be advantageous in terms of net DoF.
\end{abstract}

\section{INTRODUCTION}

Interference is a major limitation in wireless networks and the search for efficient ways of transmitting in this context has been fruitful [1]-[3]. Several techniques allow the increase of the multiplexing gain. However, most techniques rely on having accurate and instantaneous channel state information at the transmitter (CSIT) which is not realistic. CSIT can only be delayed and approximate. Though interesting results have been found concerning imperfect CSIT, the delay in the CSIT acquisition can still be an issue especially if it approaches the coherence time $T_{c}$ of the channel. The authors [4] caused a paradigm shift by proposing a scheme (MAT) reaching more than one degree of freedom (DoF) while relying solely on perfect but outdated CSIT.

MAT allows for some multiplexing gain even if the channel state changes arbitrarily over the feedback (FB) delay. The range of coherence time in which the sole use of MAT yields an increased multiplexing gain is determined in [5] and [6] but considering only FB or only training overheads and not both. We advocate for the use of a net DoF metric accounting for training overhead as well as the DoF consumption due to the FB on the reverse link to ensure fair comparison.

Since assuming independent channel variation is overly pessimistic for numerous practical scenarios, two other schemes were proposed independently in [7] and [8] for the time

\footnotetext{
$\dagger$ EURECOM's research is partially supported by its industrial members: BMW Group, iABG, Monaco Telecom, Orange, SAP, SFR, ST Microelectronics, Swisscom, Symantec, and by the EU FP7 projects NEWCOM\# and ADEL.

* Part of this work has been performed in the framework of the FP7 project ICT-317669 METIS, which is partly funded by the European Union. The authors would like to acknowledge the contributions of their colleagues in METIS, although the views expressed are those of the authors and do not necessarily represent the project.
}

correlated MISO broadcast channel with 2 users. It optimally combines delayed and current CSIT (both imperfect) but has not been generalized for a larger number of users. Another scheme that simply performs ZF and superposes MAT only during the dead times of $\mathrm{ZF}$ has been proposed in [9]. This scheme recovers the results of optimality of [7], [8] for $K=2$ and is valid for any number of users. It is based on a block fading model but it has been shown that stationary fading can be modeled exactly as a special block fading model in [10].

Even with such promising results, it was still generally believed that any delay in the FB necessarily caused a DoF loss. However, Lee and Heath in [11] proposed a scheme that achieves $N_{t}$ (sum) DoF in the block fading underdetermined MISO BC with $N_{t}$ transmit antennas and $K=N_{t}+1$ users if the FB delay is small enough $\left(T_{f b} \leq \frac{T_{c}}{K}\right)$.

If unexpected, this possibility of achieving the full sum DoF in the MISO BC with a small delay in the FB comes at the expense of a slight increase of the FB overhead. Indeed, [11] requires that, per $T_{c}, N_{t}+1$ channel states need to be fed back to achieve $N_{t}$ sum DoF. It was then demonstrated in [12] that the minimum fraction of time of perfect current CSIT required per user in order to achieve the $\operatorname{DoF}$ of $\min \left(N_{t}, K\right)$ is given by $\min \left(N_{t}, K\right) / K$. In other words, the lack of timeliness of CSIT can be compensated by having the CSIT of more users. The general results in [12] rely on having perfect current CSIT for different users at different time, which in a classic block fading model would require an increase of FB and training overheads. Similarly, [11], which is limited to the $K=N_{t}+1$ case, requires an increase of FB and training overheads [13]. In [14] this FB versus performance trade-off is characterized extensively. For the square case, i.e., when $K=N_{t}$, [14] confirms that with a block fading model and FB delay, the basic combination of MAT (when only delayed CSIT is available) and simple zero-forcing (ZF) (when current CSIT is available) is optimal in terms of DoF.

Similar efforts to find delayed CSIT schemes and bounds for the IC showed that extending BC results to multicell configurations was rarely straightforward. For the 3-user SISO IC, [15] introduces retrospective interference alignment (IA) reaching a multiplexing gain greater than one with outdated CSIT. Then in [16], a general scheme for the K-user SISO IC with outdated CSIT was shown to yield a sum DoF that is 
greater than one and increases with $K$. However, these DoF are upper bounded by $\frac{4}{6 \ln 2-1} \approx 1.266$. In [17], an ergodic interference alignment based scheme is shown to yield a DoF that increases with $K$ and approaches 2 for large $K$ in the $\mathrm{K}$-user SISO IC with outdated CSIT. There is no proof of optimality of these DoF, but it is conjectured in [16] that the DoF of the SISO IC with outdated CSIT is upper bounded by a constant. This is in sharp contrast with the optimal sum DoF of $\frac{K}{2}$ derived in [3] for the SISO IC with current CSIT. Only the result by Lee and Heath can readily be extended to the MISO IC with $N_{t}$ transmit antennas per transmitter and $K=N_{t}+1$ transmitter receiver pairs [18], in which DoF loss can be avoided for FB delay up to $\leq \frac{T_{c}}{K}$. In [19], by using a variant of ergodic interference alignment [20], the authors proves that actually DoF loss in the SISO IC can be avoided for FB delay up to $\leq \frac{T_{c}}{2}$ regardless of $K$.

The transmission schemes for the IC can be divided into two main categories. The first category, aims at approaching the DoF proper bound [21] which is not always reachable, using spatial extension, or finite time/frequency extensions. The second category is concerned with the DoF decomposition bound which is always attainable and reached by two schemes, asymptotic IA [3] and ergodic IA [20]. Depending on $K$ and $\frac{N_{t}}{N_{r}}$ either the proper bound or the decomposition bound is an upper bound on the DoF of the IC, with decomposition schemes being optimal for larger $\mathrm{K}$, when having collocated antennas no longer brings any gain.

In [22] net DoF of proper schemes were evaluated. Here we are interested in evaluating the net DoF of the two decomposition schemes, especially because of the robustness of ergodic IA to FB delay that was recently discovered and could bring net DoF gains. We succinctly review the two schemes and evaluate the net DoF they can be expected to reach in actual systems, accounting for training overhead as well as the DoF consumption due to the FB on the reverse link. For some antenna configurations, decomposition and proper bounds can be equal, therefore comparing net DoF of schemes of any category in such configuration is fair.

\section{System Model}

We first consider a K-user SISO IC, i.e., there are $\mathrm{K}$ transmitter-receiver pairs all equipped with a single antenna. Let $\mathbf{H}[t]=\left[h_{j i}(t)\right] \in \mathbb{C}^{K \times K}$ denote the channel matrix at time $t$ where $h_{j i}(t)$ is the frequency flat time-varying channel coefficient between transmitter $i$ and receiver $j$. We assume a block fading model, the channel coefficients are constant over blocks of length $T_{c}$ and change independently between blocks. Furthermore, channel coefficients are drawn from a continuous distribution, their phases are uniformly distributed and are independent from their magnitude. It is assumed that the FB is delayed but otherwise perfect.

The channel output observed at receiver $j \in[1, K]$ is a noisy linear combination of the inputs

$$
Y_{j}[t]=\sum_{i=1}^{K} h_{j i}[t] X_{i}[t]+Z_{j}[t]
$$

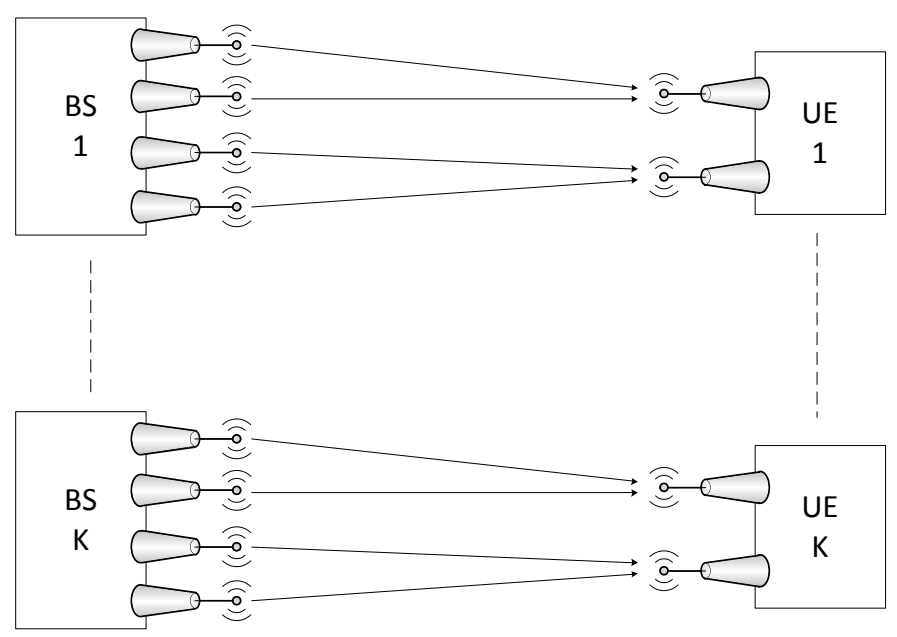

Fig. 1. Decomposition of the K user MIMO IC with $N_{t}=4$ and $N_{r}=2$, showing only links supporting intended messages.

where $X_{i}[t]$ is the transmitted symbol of transmitter $i, Z_{j}[t]$ is the additive white Gaussian noise at receiver $j$.

The first ingredient of the performance metric is the sum DoF, it is the prelog of the sum rate. Let $R_{j}(P)$ denote the achievable rate for user $j$ with transmit power $P$ then the achievable DoF for user $j$ is

$$
d_{j}=\lim _{P \rightarrow \infty} \frac{R_{j}(P)}{\log _{2}(P)}
$$

and the sum DoF of the K-user SISO IC is $\operatorname{DoF}(K)=$ $\sum_{j=1}^{K} d_{j}$.

An example of MIMO IC is shown in Fig. 1. The decomposability property is illustrated in the rectangular MIMO case in the sense that only data bearing links are shown. In the square MIMO case, with $N_{t}=N_{r}=N$, it simply means that the $K$ user MIMO IC is treated as a $K N$ user SISO IC.

\section{DECOMPOSITION SCHEMES OVERVIEW}

\section{A. Asymptotic IA}

In [3], the authors introduce the asymptotic IA that achieves $\frac{K}{2}$ DoF in the $K$ user time-varying SISO IC. It is based on the idea of interference alignment. Using symbol extension, the scheme partially aligns the interference at the receiver so that more signal dimensions can be used without interference. By using longer symbol extension, the part of non aligned symbols becomes negligible and the optimal $\frac{K}{2}$ DoF can be approached.

Precisely the scheme supports the following DoF distribution,

$$
\begin{aligned}
d_{1}(n) & =\frac{(n+1)^{N}}{(n+1)^{N}+n^{N}} \\
d_{i}(n) & =\frac{n^{N}}{(n+1)^{N}+n^{N}}, \quad i=2,3 \ldots, K
\end{aligned}
$$

with $N=(K-1)(K-2)-1$, over a $(n+1)^{N}+n^{N}$ symbol extension, thereby approaching $K / 2$ DoF as $n$ grows.

The extension of this technique to square MIMO cases is straightforward. The general MIMO case is studied in [23] in which the authors proves that one can attain $\min \left(N_{t}, N_{r}\right) K \frac{R}{R+1}$ DoF for $R=\max \left(\frac{N_{t}}{N_{r}}, \frac{N_{r}}{N_{t}}\right)$ integer. 


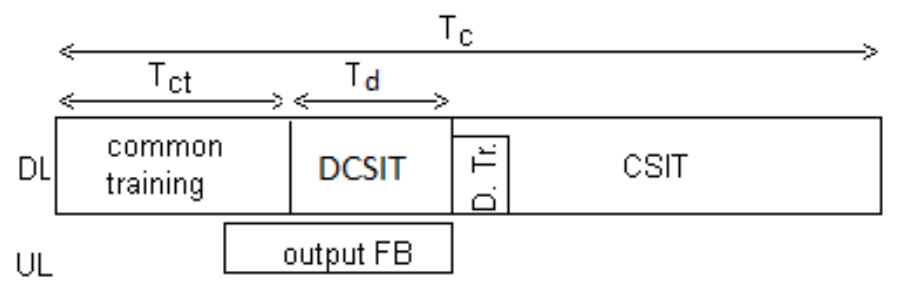

Fig. 2. Topology of a block considering the training (common and dedicated) and output FB.

The transmitters always need to have current CSI in order to make sure that the interference will be adequately aligned at the receivers.

\section{B. Ergodic IA}

The main idea behind ergodic IA is to transmit the data a first time during channel realization $\mathbf{H}\left[t_{1}\right]$, then to wait for the complementary channel realization $\mathbf{H}\left[t_{2}\right]$ such that $\mathbf{H}\left[t_{1}\right]+\mathbf{H}\left[t_{2}\right]=I$, the $K \times K$ identity matrix. We denote this relation by $\mathbf{H}\left[t_{2}\right]=\overline{\mathbf{H}\left[t_{1}\right]}$. It allows each receiver to cancel all interference by simply adding the signals received at times $t_{1}$ and $t_{2}$.

$\mathbf{H}\left[t_{2}\right]=\overline{\mathbf{H}\left[t_{1}\right]}$ cannot happen when channel coefficients are drawn from a continuous distribution. However, it is still possible to match channel matrices up to an approximation error that is small enough to allow decoding [20]. It can be done through appropriately precise quantization. The authors of [20] prove that, by considering channel realization sequences that are long enough, it is possible to be sure with a sufficient probability that it will be possible to match up enough channel realizations to yield $\mathrm{DoF}_{\text {ErgoIA }}$ that approaches $\frac{K}{2}$.

In [19], the authors notice that, in a pair of channel realizations, only the second one needs to be known by the transmitters before transmission. Hence, it is possible to approach the $\frac{K}{2}$ with delayed CSIT for one channel realization and current CSIT for the second. By modifying the channel realization pairing method the authors prove that, in a block fading model, it means that the $\frac{K}{2}$ DoF can be obtained even if the CSI acquisition takes half of the coherence time.

The extension to square MIMO cases is straightforward. For rectangular cases, the results of asymptotic IA can be attained, i.e., one can attain $\min \left(N_{t}, N_{r}\right) K \frac{R}{R+1}$ DoF and the pairing schemes takes $R+1$ channel uses but only necessitate current CSIT for the last $R$ therefore these DoF are attainable even if the CSI acquisition takes $\frac{1}{R+1}$ of the coherence time [24].

\section{Decomposability}

The ergodic IA and asymptotic IA schemes do not require any joint antenna processing and can therefore also be used in interfering broadcast channels (IBC) and interfering multiple access channels (IMAC). Then, for instance in a IBC with $K$ cells, $K_{b}$ receivers per cell, $N_{t}$ per transmitter, $N_{r}$ antennas per receiver, the attainable DoF is the same as in the IC with $K$ cells, $N_{t}$ antennas per transmitter, $K_{b} N_{r}$ antennas per receiver.

\section{NET DOF CHARACTERIZATION}

In order to compare the multiplexing gains that asymptotic IA and ergodic IA can be expected to obtain in actual systems, we derive their net DoFs, accounting for training and FB overhead. In other words we evaluate how many DoF are available for data on the forward link (we account for delay and training) and subtract the DoF wasted on the reverse link for the FB.

\section{A. CSI Acquisition Overheads}

For the $K$ Rxs to estimate their channel, a common training of length greater than or equal to $N_{t}$ per Tx is needed, resulting in a total training length $T_{c t} \geq K N_{t}$. To maximize the DoF we take the minimal $T_{c t}=K N_{t}$. According to [25], an additional dedicated training of $d_{k}$ pilot is required in the end, to assure coherent reception at receiver $k$, resulting in $T_{t r_{a}}=K N_{t}+$ $\sum_{k} d_{k}$ symbol periods per block devoted to training in order to perform asymptotic IA. For ergodic IA, the only difference is that there is no need for dedicated training as no precoding is done resulting in $T_{t r_{e}}=K N_{t}$ symbol periods per block devoted to training in order to perform ergodic IA.

Since we are interested in the DoF consumed by the FB, which is the scaling of the FB rate with $\log _{2}(P)$ as $P \rightarrow \infty$, the noise in the fed back channel estimate can be ignored in the case of analog FB or of digital FB of equivalent rate. The FB can be considered accurate, suffering only from the delay. We consider analog FB and two FB strategies. First, channel feedback (CFB), the RXs estimate the channel state from the training sequences and feed back their channel estimate. Second, output feedback (OFB), the Rxs directly feed back the training signals they receive and the Txs perform the (downlink) channel estimation. User $k$ needs to feedback the coefficients of its $K$ channels with Tx $i, i \neq k$, i.e.; $K N_{t} N_{r}$ coefficient to feedback per user. The total FB is $K K N_{t} N_{r}$ symbols and consumes $T_{F B_{a}}=K K N_{r}$ channel uses on the reverse link for both feedback strategies to do asymptotic IA.

A slight improvement could be made in case of ergodic IA. As was mentioned in [26], there exist an optimal pairing that maximizes the SNR offset which imposes a relation between coefficients of direct links, $\mathbf{H}\left[t_{1}\right](k, k)$ and $\mathbf{H}\left[t_{2}\right](k, k)$, in the two channel realizations. However, for DoF purposes, we only requires that coefficients of direct links are not additive inverses so that the intended signal is not canceled when received signals are added. Therefore one bit feedback for the direct links is enough to do the pairing and it is null in terms of DoF so the FB cost can be reduced to $T_{F B_{e}}=K(K-1) N_{r}$ channel uses for ergodic IA.

The difference between CFB and OFB is the time it takes for the TX to have CSI after the training is done, with CFB it takes $T_{d, C F B}=T_{F B}+T_{f d}$ where $T_{f d}$ is the delay in the FB due to processing and propagation. With OFB the Rxs do not have to wait for all the training to be done to start the $\mathrm{FB}$ and we have $T_{d, O F B}=\max \left(T_{F B}+T_{f d}-T_{t r}, T_{f d}\right)$ as it cannot be less than $T_{f d}$. In order to have only one expression for the netDoF we will use the following notation, $T_{d_{a}}$ and $T_{d_{e}}$, the delayed CSIT (DCSIT) time. It is the total time between the end of training and the moment CSI available at the transmitters which will be equal to $T_{d, C F B}$ or $T_{d, O F B}$ depending on the FB strategy. In 
other words it corresponds to the time spent with the forward link being free but with the transmitter not having CSI yet.

These FB length values are obtained assuming a distributed model, each Tx gets all the CSI from FB without the need for a central unit. In Fig. IV an illustration of a block is given for a better understanding of the different parts. The two parts available for downlink transmission are DCSIT and CSIT, they respectively correspond to the transmitter having only past CSI and past CSIT together with current CSI.

For more elaborate derivations of the net Dof see [9], [10], [22].

\section{B. Asymptotic IA}

Whit current CSIT, the full multiplexing gain can be achieved with asymptotic IA. Doing only asymptotic IA would allow to transmit an average of $D=\min \left(N_{t}, N_{r}\right) \frac{R}{R+1}$ symbols per channel use to each user when the transmitter has CSIT and nothing otherwise (dead time). Taking FB and training into account we obtain

$$
\begin{aligned}
& \text { netDoF }(\text { aIA })=D\left(1-\frac{T_{\text {delay }_{a}}+K K N_{r}}{T_{c}}\right) \\
& =D\left(1-\frac{\overbrace{T_{d_{a}}}^{\text {dead time }}+\overbrace{K N_{t}+D}^{\text {training }}+\overbrace{K K N_{r}}^{\mathrm{FB}}}{T_{c}}\right)
\end{aligned}
$$

where $T_{\text {delay }_{a}}=T_{d_{a}}+T_{t r_{a}}$ is the CSIT acquisition delay.

\section{Ergodic IA}

Whit current CSIT, the full multiplexing gain can be achieved with ergodic IA. The difference is that ergodic IA can also be performed over the DCSIT time $T_{d_{e}}$ as long as it is not longer than the time with current CSIT [19], i.e., less than $T_{c}-T_{\text {delay }_{e}}$. Taking FB and training into account we obtain

$$
D\left(1-\frac{\overbrace{\left(T_{d_{e}}-\left(T_{c}-T_{\text {delay }_{e}}\right)\right)^{+}}^{\text {dead time }}+\overbrace{K N_{t}}^{\text {training }}+\overbrace{K(K-1) N_{r}}^{\text {FB }}}{T_{c}}\right)
$$

indeed $T_{c}-T_{\text {delay }_{e}}$ is the number of channel uses available for transmission with CSIT and, with $(a)^{+}$denoting $\max (0, a)$, $\left(T_{d_{e}}-\left(T_{c}-T_{\text {delay }_{e}}\right)\right)^{+}$is the part of DCSIT time that cannot be used with ergodic IA and is actually lost (dead time).

\section{TDMA-IA}

Time division multiple access (TDMA) gives $\min \left(N_{t}, N_{r}\right)$ DoF and only require CSI at the receiver, which is available during the DCSIT time since the training has already been done. Therefore, doing TDMA over the DCSIT time that is not yet used is a simple way to improve the net DoF of asymptotic IA, and possibly of ergodic IA in case all the DCSIT time cannot be used to perform ergodic IA. We obtain

$\operatorname{netDoF}($ TDMA-aIA $)=\operatorname{netDoF}($ aIA $)+\frac{\min \left(N_{t}, N_{r}\right) T_{d_{e}}}{T_{c}}$

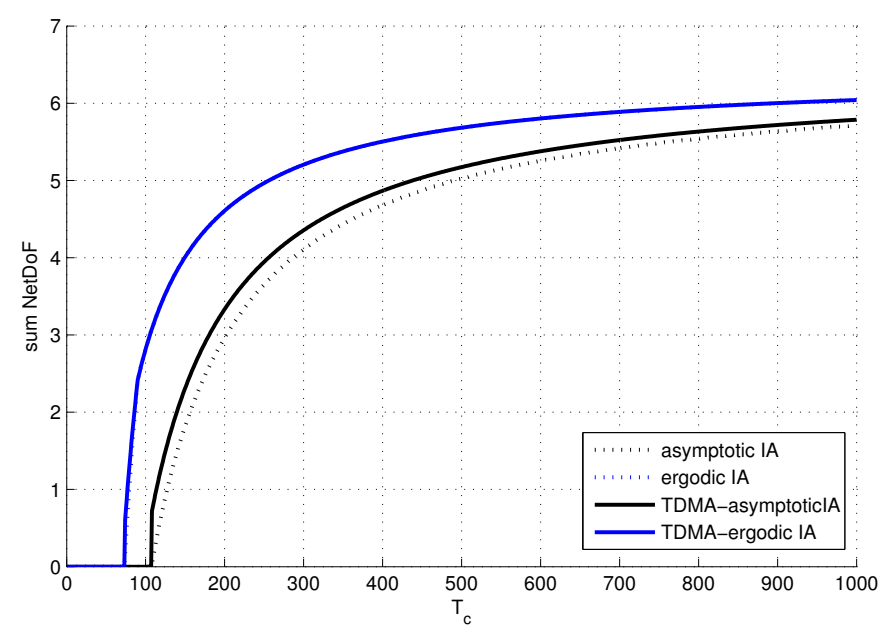

Fig. 3. NetDoF of asymptotic IA, ergodic IA and their combination with TDMA for $K=4, N_{t}=8, N_{r}=2, T_{f d}=3$ as a function of $T_{c}$.

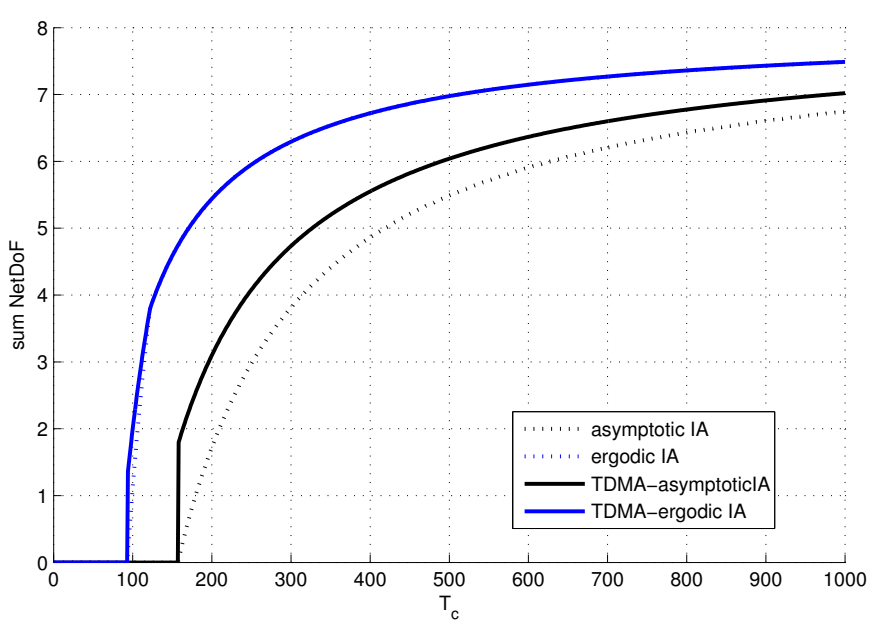

Fig. 4. NetDoF of asymptotic IA, ergodic IA and their combination with TDMA for $K=4, N_{t}=N_{r}=4, T_{f d}=3$ as a function of $T_{c}$.

and

$$
\begin{aligned}
& \text { netDoF(TDMA-eIA })=\operatorname{netDoF}(\mathrm{eIA})+ \\
& \qquad \frac{\min \left(N_{t}, N_{r}\right)\left(T_{d}-\left(T_{c}-T_{\text {delay }_{e}}\right)\right)^{+}}{T_{c}}
\end{aligned}
$$

More elaborate schemes could be used to benefit from the DCSIT time when TDMA could actually work even with no CSIT at all. However, unlike in the BC, the DCSIT schemes for IC have showed limited gain so far.

\section{E. Numerical results}

In Fig. 3 we plot the netDoF provided by asymptotic IA, ergodic IA and their combination with TDMA for $K=4$, $N_{t}=8, N_{r}=2, T_{f d}=3$ as a function of $T_{c}$, using (3) for asymptotic IA, (4) for ergodic IA, (5) for TDMAasmyptotic IA and (6) for TDMA-ergodic IA. OFB was chosen as it reduces the FB delay. We notice that asymptotic IA is improved by the addition of TDMA but still is largely outperformed by ergodic IA, because the ergodic IA variant loses almost no DoF to FB delay and does not require extra overheads. Since the DCSIT time is most of the time used to perform ergodic IA, the addition of TDMA is not significant. 


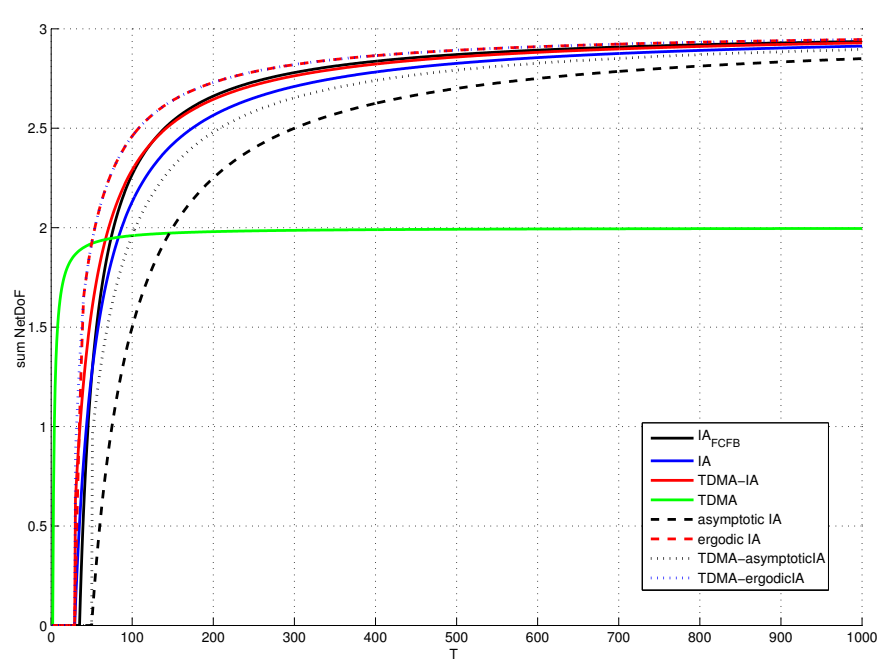

Fig. 5. NetDoF comparison of decomposition schemes and linear schemes for $K=3, N_{t}=N_{r}=2, T_{f d}=3$ as a function of $T_{c}$.

In Fig. 4 the same observations can be made, this time in a square MIMO IC with $K=4, N_{t}=N_{r}=4, T_{f d}=3$.

In Fig. 5 we compare the netDoF of the decomposition schemes with proper schemes. To be fair, we consider a configuration where the proper bound and the decomposition bound are both equal to 1 DoF per user: $K=3$ and $N_{t}=N_{r}=2$. We notice that asymptotic IA has similar performances as the proper schemes, this is because it has the same DoF and similar losses due to FB delay. On the contrary, thanks to its robustness to FB delay, ergodic IA outperforms all the others schemes as soon as simple TDMA is not optimal anymore. However, it is worth mentioning that both asymptotic and ergodic IA induce similarly long decoding delays to approach the decomposition bound in comparison with proper schemes.

As mentioned in [22], the numbers of active cells and active antennas $N_{t}$ and $N_{r}$ need to be optimized to find the right channel learning/using compromise because serving more users (or having more active antennas) means a larger DoF but also larger overheads. This why for small $T_{c}$, TDMA, i.e., single user MIMO, is optimal.

\section{CONCLUSION}

Ergodic IA and asymptotic IA have a few similarities, they reach the decomposition bound, they are decomposable and they induce large decoding delays. In this paper however, we show that, thanks to its variant, robust to FB delays, ergodic IA attains significantly larger net DoF than asymptotic IA especially for small channel coherence time. Moreover, when they yield the same DoF, ergodic IA also outperforms the proper schemes in net DoF.

\section{REFERENCES}

[1] G. Caire and S. Shamai, "On the achievable throughput of a multiantenna gaussian broadcast channel," IEEE Transactions on Information Theory, vol. 49, no. 7, pp. 1691-1706, July 2003.

[2] D. Gesbert, S. Hanly, H. Huang, S. Shamai Shitz, O. Simeone, and W. Yu, "Multi-cell mimo cooperative networks: A new look at interference," IEEE Journal on Selected Areas in Communications, vol. 28, no. 9 , pp. $1380-1408$, december 2010 .
[3] V. Cadambe and S. Jafar, "Interference alignment and degrees of freedom of the k-user interference channel," IEEE Transactions on Information Theory, vol. 54, no. 8, pp. 3425 -3441, aug. 2008.

[4] M. Maddah-Ali and D. Tse, "Completely stale transmitter channel state information is still very useful," in Proc. Allerton, Monticello, IL, USA, Oct. 2010.

[5] J. Xu, J. Andrews, and S. Jafar, "Miso broadcast channels with delayed finite-rate feedback: Predict or observe?" IEEE Transactions on Wireless Communications, vol. 11, no. 4, pp. 1456-1467, 2012.

[6] M. Kobayashi and G. Caire, "On the net DoF comparison between ZF and MAT over time-varying MISO broadcast channels," in Proc. ISIT, Boston, MA, USA, Jun. 2012.

[7] T. Gou and S. Jafar, "Optimal use of current and outdated channel state information: Degrees of freedom of the MISO BC with mixed CSIT," IEEE Communications Letters, vol. 16, no. 7, pp. 1084-1087, Jul. 2012.

[8] S. Yang, M. Kobayashi, D. Gesbert, and X. Yi, "Degrees of freedom of time correlated MISO broadcast channel with delayed CSIT," IEEE Transactions on Information Theory, vol. 59, no. 1, pp. 315-328, Jan. 2013.

[9] Y. Lejosne, D. Slock, and Y. Yuan-Wu, " Degrees of freedom in the MISO BC with delayed-CSIT and finite coherence time: a simple optimal scheme," in Proc. ICSPCC, Hong Kong, China, Aug. 2012.

[10] , "Finite rate of innovation channel models and DoF of MIMO multi-user systems with delayed CSIT feedback," in Proc. ITA, San Diego, CA, USA, Feb. 2013.

[11] N. Lee and R. W. Heath Jr., "Not too delayed csit achieves the optimal degrees of freedom," in Proc. Allerton, Monticello, IL, USA, Oct. 2012.

[12] R. Tandon, S. A. Jafar, and S. Shamai, "Minimum CSIT to achieve maximum degrees of freedom for the MISO BC," CoRR, vol. abs/1211.4254, 2012, http://arxiv.org/abs/1211.4254.

[13] Y. Lejosne, D. Slock, and Y. Yuan-Wu, "Net degrees of freedom of recent schemes for the MISO BC with delayed CSIT and finite coherence time," in Proc. WCNC, Shanghai, China, Apr. 2013.

[14] J. Chen, S. Yang, and P. Elia, "On the fundamental feedback-vsperformance tradeoff over the miso-bc with imperfect and delayed csit," in Proc. ISIT, Istanbul, Turkey, Jul. 2013.

[15] H. Maleki, S. Jafar, and S. Shamai, "Retrospective interference alignment," in Proc. ISIT, St Petersburg, Russia, Aug. 2011.

[16] M. J. Abdoli, A. Ghasemi, and A. K. Khandani, "On the degrees of freedom of K-user SISO interference and X channels with delayed CSIT," Submitted to IEEE Transactions on Information Theory, vol. abs/1109.4314, Sep. 2011, http://arxiv.org/abs/1109.4314.

[17] M. Kang and W. Choi, "Ergodic interference alignment with delayed feedback," IEEE Signal Process. Lett., no. 5, 2013.

[18] N. Lee and R. W. Heath Jr., "CSI feedback delay and degrees of freedom gain trade-off for the MISO interference channel," in Proc. Asilomar, Pacific Grove, CA, USA, Nov. 2012.

[19] Y. Lejosne, D. Slock, and Y. Yuan-Wu, "Achieving full sum dof in the SISO interference channel with feedback delay," to appear in IEEE Com. Lett., 2014.

[20] B. Nazer, M. Gastpar, S. Jafar, and S. Vishwanath, "Ergodic interference alignment," IEEE Transactions on Information Theory, vol. 58, no. 10, pp. 6355-6371, Oct. 2012.

[21] C. Yetis, T. Gou, S. Jafar, and A. Kayran, "On feasibility of interference alignment in mimo interference networks," IEEE Transactions on Signal Processing, no. 9, 2010.

[22] Y. Lejosne, D. Slock, and Y. Yuan-Wu, " Foresighted delayed CSIT feedback for finite rate of innovation channel models and attainable netDoFs of the MIMO interference channel," in Proc. Wireless Days (WD), Valencia, Spain, Nov. 2013.

[23] T. Gou and S. Jafar, "Degrees of freedom of the k user MxN MIMO interference channel," IEEE Transactions on Information Theory, vol. 56, no. 12, pp. 6040-6057, Dec. 2010.

[24] Y. Lejosne, D. Slock, and Y. Yuan-Wu, "Ergodic interference alignment for the SIMO/MIMO interference channel," in Proc. ICASSP, Firenze, Italy, May 2014.

[25] F. Negro, D. Slock, and I. Ghauri, "On the noisy MIMO interference channel with CSI through analog feedback," in Proc. ISCCSP, Rome, Italy, May 2012.

[26] C. Geng and S. Jafar, "On optimal ergodic interference alignment," in Proc. GLOBECOM, Anaheim, CA, USA, Dec. 2012. 